\title{
MARY SHELLEY: IL PADRE COME OSSESSIVA RELAZIONE
}

MARY SHELLEY: THE FATHER AS AN OBSSIVE RELATIONSHIP

Clelia Stefanuto

\section{Riassunto:}

L'obiettivo di questo articolo è analizzare le difficili relazioni paterno-filiali in Frankenstein di Mary Shelley, contestualizzando l'opera nella narrativa dell'Ottocento nel quale si tenta di ridefinire il ruolo della figura del padre nella famiglia e nella società. Mary Shelley proietta nel mostro il suo rapporto conflittuale con Godwin creando un racconto obliquamente autobiografico e, allo stesso tempo, emblematico della ribellione delle figlie e scrittrici al criterio di autorità patriarcale.

\section{Palabras clave:}

relazioni padre-figlia, Frankenstein, Mary Shelley

\section{Abstract:}

The aim of this article is to analyze the difficult paternal-filial relationships in Mary Shelley's Frankenstein, contextualizing the work in nineteenth-century fiction in which an attempt is made to redefine the role of the father figure in the family and in society. Mary Shelley projects her conflictual relationship with Godwin into the monster, creating an obliquely autobiographical tale and, at the same time, emblematic of the rebellion of her daughters and writers against the criterion of patriarchal authority.

\section{KEYWORDS:}

relationship father-daughter, Frankenstein, Mary Shelley 


\section{Contro la figura del padre}

La rivoluzione francese (1789-1795) come appunta Margaret Darrow "trasformò il tempo in una nuova era, lo spazio in nuove misure, l'identità sociale in una nuova ottica (citoyen) e l'identità personale in una moltitudine di nuovi nomi (..) Questo nuovo panorama, non lascia invariata nemmeno la famiglia tradizionale, che viene distrutta per essere ricostruita" (Darrow, 1989: 11).

Il grande romanzo dell'Ottocento europeo è la testimonianza della messa in discussione delle relazioni tra i sessi e l'organizzazione della famiglia che, dopo la grande rivoluzione, si impegnò nella ridefinizione dei rapporti che intercorrono tra "stato" e "cittadinanza", stabilendo come punto di partenza le relazioni familiari. Il dibattito sulla concezione di "famiglia" crea una frattura tra liberali e conservatori. Entrambi, però, si trovano d'accordo nel dichiarare che, per essere tale, debba costruire le sue fondamenta su una struttura patriarcale, la quale confina le donne nello spazio privato della casa.

Gli intellettuali del tempo si concentrarono sulla "ricostruzione del padre" inteso come modello democratico dai liberali e come autorità monarchica dai tradizionalisti. Tra i romanzi del tempo troviamo grandi opere che portano la firma di de Balzac, Dickens, e Miller, autori che propongono una lettura paterna che rimpiange il modello dell'ancien regime, caratterizzato da un simbolismo prettamente patriarcale: Padre, Re e Dio. Oltre all'esaltazione del modello patriarcale è fortemente presente la critica diretta al femminismo e all'ipotesi "spaventosa" che le donne possano prendere il sopravvento ed estirpare l'uomo dalla sua posizione di comando; Papà Goriot (1834) ne è un chiaro esempio. La produzione femminile ottocentesca, rispetto al Settecento, si occupa di figlie che, seppur continuino a caricarsi di "colpe", appaiono nel complesso meno idealizzate e più inclini al sacrificio. Sulle orme di Corinna (1807) di Madame de Stael, iniziano a prende forma, tra le pagine delle grandi opere femminili, nuovi personaggi che rappresentano l'embrione delle future figlie ribelli, le quali abitano lo spazio situato tra il desiderio di riconoscimento e l'impegno in un self-fashioning privo di autorità paterna.

Le scrittrici dell' 800 , di fronte alla crisi e alla trasformazione dell'autorità nella società ottocentesca, presero parte al panorama letterario in maniera vivace, contribuendo alla produzione dei romanzi "formativi" del tempo, creando così un nuovo spazio, una nuova opportunità d'espressione. Le scrittrici rappresentano la figura dell'androgino e la società dell'Ottocento non è ancora pronta ad accettare la sovrapposizione tra le categorie "donna" e "scrittrice". William Veeder, nella sua introduzione a Mary Shelley and Frankenstein, illustra questa situazione: "Mary Shelley's novels [ ...] reflect her lifelong concern with the psychological ideal of androgyny and its opposite, 
bifurcation-the harmonious balance of traits traditionally considered masculine and feminine, and the desolating polarization of these traits" (Veeder, 1986: 2).

La domesticità come ideologia forgia stereotipi come quello dell'angelo del focolare (Kirkpatrick, 1989), contro il quale si alzano le opere di scrittrici come Mary Wollstonecraft, Mary Shelley e Jane Austen. Gilbert e Gubar sostengono che la presenza delle scrittrici, donne che svolgono la loro professione in un ambito pubblico, è vista come qualcosa di anomalo "a woman writer does not 'fit in'...she seems to be anomalous, indefinable, alienated, a freakish outsider" (Gilbert e Gubar, 1979: 48). Se a questo si aggiunge la mancanza di riferimenti e modelli femminili nella letteratura, si può affermare che le scrittrici irrompono nella scrittura confrontandosi con una tradizione maschile che rappresenta l'autorità patriarcale. In questo senso possiamo leggere le opere di molte scrittrici, inclusa Mary Shelly, come scrittura di ribellione contro la figura del padre.

\section{Mary Shelley. Il Padre OMnipresente}

L'interesse generale per il tema delle relazioni paterno-filiali appare ancor più marcato quando a parlare del padre, sono scrittrici prive di una figura materna, come Mary Shelley.

Figlia del filosofo radicale William Godwin e di Mary Wollstonecraft, coraggiosa promotrice dei diritti delle donne, scrittrice ed intellettuale, morta di parto il 10 settembre 1797, Mary Shelley si forma in un ambiente interamente dominato dalla figura paterna. L'assenza del materno e la totale identificazione con il padre, che per primo ammette la sua incapacità per occuparsi delle figlie: "Quelle povere bambine! Sono completamente inetto a educarle (...). Sono la persona meno adatta a svolgere questo compito" (Brown, 1926. 78), propiziano un'identificazione della scrittrice con l'archetipo della "donna senza madre" ${ }^{1}$. La cancellazione dell'eredità materna, non soltanto in termini affettivi, ma soprattutto intellettuali significò per Mary l'impossibilità di ricevere quell'educazione in uguaglianza che sua madre aveva teorizzato nel suo Vendicazione dei diritti delle donne (Wollstonecraft, 1792). L'assenza dell'educazione paterna la porta all'autodidattismo. Se la figura della madre scomparsa, fu il motivo principale per cui si dedicò integramente alla scrittura ed alla lettura, "Especialy because she never know her mother" (Gilbert, 1978: 65), la biblioteca del padre le proporzionò tutte le letture necessarie per conoscere gli autori fondamentali per l'elaborazione della sua opera.

1 Rappresentato da Atena, dea della saggezza e dei mestieri, uscita dalla testa di Zeus con l'aiuto di Efeso, il quale ruppe la testa del Dio con un'ascia a doppio taglio, favorendo il parto. Metis, la madre di Atena, venne inghiottita da Zeus, il quale divenne l'unico referente della dea greca, l'unico modello con il quale si identificherà completamente. Nel caso di Mary Shelley, come sostiene Bolen, "Non tutte le donne Atena hanno un padre Zeus che le appoggia. In questo caso, manca un ingrediente essenziale allo sviluppo. Alcune hanno un padre assai in vista, troppo impegnato per notarle" (Bolen, 1991: 76). 
Fin dall'infanzia, il rapporto tra Mary Shelley e il padre si rivelò problematico, ed in qualche modo ambivalente. La prima grande frattura tra Mary e Godwin avvenne dopo il matrimonio di quest'ultimo con la loro vicina di casa, Mary Jane Clairmont, vedova e già madre di due figli, con la quale Mary si scontrerà per tutta la vita (Praz, 2011). L'adolescenza della scrittrice fu largamente influenzata dall'ambiente domestico, dove ebbe la possibilità di usufruire della grande biblioteca di famiglia e dove incontrò molti intellettuali dell'epoca, i quali frequentavano assiduamente la famiglia Godwin. Nonostante la forte influenza che ebbe sulla scrittrice l'ambiente familiare, Mary trascorse poco tempo in casa, poiché il padre l'allontanò per ben due volte: nel 1811, quando venne mandata a Ramsgate, nel Kent, e nel 1813, quando venne mandata in Scozia. L'atteggiamento freddo e distaccato del padre si evince soprattutto nella corrispondenza che ebbe con la seconda moglie, quando, nell'estate del 1811, la salute di Mary fu messa a rischio da un terribile eczema del braccio. Godwin, in questa circostanza, scrive alla consorte: “Di' a Mary che, malgrado le apparenze sfavorevoli, confido ancora che diventerà una donna assennata e, per di più, buona e felice" ${ }^{\prime 2}$. Più che un messaggio preoccupato, è evidentemente un augurio che presenta un grande distacco emotivo. Godwin attraverso le lettere di diario della figlia e gli appunti del suo diario, risulta essere da un lato un padre distaccato e freddo, perfettamente in linea con l'ideale patriarcale del tempo, il quale da un lato considerava la figlia "una sorta di proprietà", dall'altro invece è evidente che Godwin vede la figlia semplicemente come lo stereotipo di "donna scrittrice" una sorta di essere androgino, da temere e da tenere alla larga. È proprio questo forse, il motivo per il quale Godwin alternò sempre un atteggiamento distaccato e disinteressato ad un atteggiamento possessivo e soffocante nei confronti della figlia.

I rapporti tra il padre e la scrittrice si incrinarono ulteriormente a causa del legame di Mary con Percy Shelley, il giovane poeta conosciuto proprio in casa Godwin. Percy aveva incominciato a frequentare la famiglia per via della fervente adesione alle idee libertine di Godwin, espresse dal poeta soprattutto nella celebre opera Political Justice (1793). Appare infatti molto incoerente e arbitrario il dissenso paterno nei confronti di questo legame. Shelley era sposato con la sedicenne Harriet Westbrook, ma sulla base delle dichiarazioni espresse da Godwin in merito alla vacuità del contratto matrimoniale o alla libertà insita nel rapporto amoroso, tale elemento non avrebbe dovuto rappresentare un impedimento per l'unione tra Mary e Percy. Godwin invece, considerò oltraggioso il legame della figlia con un uomo sposato, e recise nettamente ogni rapporto con Mary nel momento in cui la figlia fuggì in Francia con Shelley, nel 1814.

2 http://www.bodley.ox.ac.uk/dept/scwmss/wmss/online/1500 
L'allontanamento paterno contribuì sicuramente a modificare il legame tra Shelley e la scrittrice, facendo sì che il compagno divenisse per Mary una sorta di surrogato di Godwin. Il rapporto paterno aveva insegnato alla scrittrice a confondere "affetto" con "approvazione" e "amore incondizionato" con il suo contrario. In una lettera del 1814 la scrittrice confessa a Shelley: "Perché Godwin non segue la naturale inclinazione dei suoi affetti e non si riconcilia con noi. (...) Buona notte, amore mio (...) tieni stretta al cuore la tua Mary. Forse, un giorno, anche lei avrà un padre; fino ad allora, sii tutto per me amore e io farò la brava (...) imparerò il greco (...) e tu potrai ricompensarmi con dolcezza" (Brown, 1926: 45).

La ricompensa intesa da Mary potrebbe essere di tipo sessuale, ma è inequivocabilmente un premio per essersi comportata bene. La scrittrice sembra incarnare il consueto rovesciamento freudiano: aveva conquistato e sedotto il padre con l'intelligenza e, da giovane donna, cercava di suscitare amore e approvazione allo stesso modo. La scrittrice ribadisce il concetto di "amore meritato" anche tra le righe di Frankenstein, quando Victor parlando del padre ammette che: "Nella mente retta di mio padre albergava un senso di giustizia che gli rendeva indispensabile stimare immensamente per amare immensamente" (Shelley, 2019: 59). Anche quando il dottor Frankenstein, pensando al suo successo come scienziato, dichiara: "Nessun padre avrebbe mai potuto rivendicare da parte del figlio una gratitudine così assoluta come quella che avrei meritato io da loro" (Shelley, 2019: 82). O ancora, quando lo scienziato si rivolge all'amico Clerval dicendogli: "come puoi immaginare che il mio primo pensiero non sia volato verso i miei cari, che tanto amo e che meritano tanto il mio amore?" (Shelley, 2019: 92).

Malgrado il distacco emotivo di Godwin nei confronti della figlia, il poeta vedeva in lei la sua discepola diretta, ed è proprio questo l'elemento che rende ambivalente il rapporto tra i due. Da un lato il padre la considera una "degna erede", dall'altro invece, la femminilità della figlia rappresenta uno scoglio enorme. Da sempre Mary manifestò un forte interesse verso la scrittura, intravedendo in essa il mezzo che le avrebbe consentito di conoscere la madre (attraverso la lettura delle sue opere) e grazie al quale avrebbe potuto reputarsi degna di portare il nome di due grandi scrittori. Il processo creativo venne visto dalla scrittrice come una prova "per mostrarsi all'altezza" dei suoi genitori, ma il risultato di questo sforzo tardò ad arrivare. La prima opera dell'autrice esce nel 1818, dopo due anni di lavoro e varie complicazioni riscontrate nella pubblicazione ${ }^{3}$, e forse è proprio a causa della lunga e sofferta attesa

3 Mary Shelley compone l'opera tra il 1816 e il 1817, anni in cui la scrittrice dovette affrontare due morti, un matrimonio e una nascita, facendo sì che la stesura del testo fosse particolarmente tormentata. Il testo subì moltissime variazioni e riscritture prima di giungere alla data della pubblicazione e nei taccuini sono presenti spesso le correzioni messe in atto dal marito Percy. Anche il processo di pubblicazione apparve complesso: né Godwin né Percy riuscirono a far pubblicare il romanzo velocemente presso una casa editrice a causa della sua portata bizzarra, così che Mary, 
che Frankenstein presenta alcuni tratti che lo rendono quello che poi la stessa Shelley chiamerà "my hideous progeny". Già questa precisazione porta certamente a dire che in Frankenstein la figura paterna è dominante e appare non soltanto nella figura del professore, ma in molti altri personaggi, tutti collegati tra loro dalla condizione di disubbidienza, elemento che sicuramente si ricollega anche all'esperienza personale dell'autrice coerentemente con il dissenso paterno nei confronti della relazione con Percy.

\section{FrankNESTEIN, L'OMbRA DEL PADRE}

Il padre di Mary č il primo destinatario della prima edizione di Frankenstein (1818), al quale č dedicato il libro. Ma anche la figura del marito ha un ruolo importante in questa prima edizione poiché apporta alcune correzioni e scrive il prologo dell'opera. La prima edizione fu pubblicata anonimamente e fu subito attribuita al padre: "Presumably because it was unthinkable that a woman should refuse to moralize, most critics automatically assumed that the author of Frankenstein was a man" (Poovey, 1987: 83). Si ipotizzò anche che l'opera fosse stata scritta da Percy Shelley, suo marito. Steven Forry (1990), sottolinea il successo immediato del romanzo, la cui prima edizione fu adattata al teatro da Richard Peake, nella famosa versione del 1831: Presumption; or The Fate of Frankenstein, alla cui popolarità si deve secondo lui, la terza edizione del romanzo, pubblicata lo stesso anno.

Nella terza edizione di Frankenstein (1831), Mary prende le redini del suo testo, affrancandosi da queste due figure maschili. La revisione del suo testo mira a guadagnare-consolidare una posizione sociale che possa costituire un' eredità per suo figlio Percy Florence. Questo dimostra una complessa strategia retorica in propria difesa, sostenendo i motivi che l'hanno spinta a scrivere l'opera. La Shelley descrive la sua gioventù e sostiene che non era sua intenzione né abbandonare i doveri da "donna", né invidiare la fama degli scrittori uomini, e fa cadere la responsabilità della stesura dell'opera su due personaggi maschili, sostenendo che la "obbligarono" a scrivere.

Prendendo in esame le tre edizioni nel loro insieme, l'opera di Frankenstein rappresenta un caso di studio inerente al confronto delle aspettative che la Shelley associava da una parte a sua madre e all'originalità romantica, dall'altra al fatto che voleva rispondere in maniera chiara all'ideale di convertirsi in una dama (Poovey, 1987). Le tre versioni sono molto significative per quanto riguarda lo studio dell'immagine del padre: Mary passa dalla posizione di figlia, che si rapporta alla figura paterna o

da brava figlia di editore, capì che prima di Frankenstein doveva pubblicare un libro che avrebbe riscosso successo da parte di un pubblico più ampio possibile: scrisse così in forma di romanzo il suo Diario dove parlava della fuga di amore con Percy, che fu immediatamente pubblicato da Hookham e Ollier. Dopo il suo esordio, proprio come aveva previsto, pochissimi mesi dopo le case editrici accettarono di pubblicare il manoscritto di Frankenstein. 
alla figura del marito, sostitutiva di esso, a quella di madre che costruisce il futuro del proprio figlio.

Il suo matrimonio, comunque, in qualche modo rappresenta la resa della scrittrice: Godwin nonostante fosse un grande sostenitore del rapporto d'amore svincolato dal matrimonio (Godwin, 1794) tagliò ogni rapporto con la figlia all'ufficializzazione del suo rapporto con Percy, poiché, quando si trattava della figlia, il concetto patriarcale di "oggetto di scambio" aveva la meglio su qualsiasi idea liberale.

Una parte della critica, alla luce di alcuni elementi della vita di Mary Shelley, ha sottolineato il carattere obliquamente autobiografico del personaggio di Frankenstein. Anche Barabara Johnson afferma che il suo personaggio "can be read in the story of autobiography as the attempt to neutralize the monstrosity of autobiography" (Johnson, 1987: 58).

Gilbert e Gubar (1979), sostengono che le scrittrici dell'epoca vittoriana in Inghilterra si identificano nella figura del mostro. In contrapposizione alla donna virtuosa, "angelo del focolare" che svolge la sua vita nella domesticità della sfera privata, la scrittrice è percepita dalla società come una minaccia, la sua invasione illecita nel campo dell'immaginazione e della creazione, deturpano la sua femminilità, che diventa incontrollabile e fa di lei qualcosa di mostruoso. La figura del mostro diventa la metafora dell'incomodità delle scrittrici per il ruolo sociale a loro assegnato. La creatura non è il doppio perfetto della sua autrice, ma costituisce una figura dell'ansietà nella costruzione di un' identità che allo stesso tempo si sottomette e si ribella, accetta gli stereotipi sociali e tenta di costruire uno spazio alternativo dal quale poter parlare. Elisabetta Marino nel suo saggio lo ribadisce in questa maniera:

Disubbidire al genitore è condizione necessaria acciocché il figlio possa trasformarsi in individuo; ed ecco che i protagonisti maschili del romanzo contravvengono costantemente alle indicazioni date loro dai padri, decretando, il più delle volte, la propria rovina. Walton (...) intraprende la via del mare che era stata proibita; Clerval (...) continua i suoi studi sebbene la tradizione familiare voglia che lui si realizzi nel commercio, lo scienziato stesso nutre un interesse profondo per gli scritti di Agrippa e Paracelso (...) suscitando le ire del padre (Marino, 2009: 79).

Il padre del dottor Frankenstein è un uomo buono, e in molti passi del libro è deducibile che l'amore che prova nei confronti del figlio è un amore sincero e profondo. È interessante notare come all'interno della narrazione venga descritto come colui che indirizza Victor verso la conoscenza, proprio come fece Godwin con Mary: "Nell'impartirmi la sua educazione mio padre aveva preso le massime precauzioni per evitare di imprimere nella mia mente qualunque forma di terrore soprannaturale" (Shelley, 2019: 80). 
Dalla lettura dell'opera, il rapporto tra il dottor Frankenstein e il padre viene descritto come un legame fondato sulla stima, sull'amore e sul rispetto: "Amato e venerabile genitore! Mi rimaneva ancora lui" (Shelley, 2019: 80). Il padre che educa, il padre che rifiuta, il figlio che ama il genitore e quello che lo odia. Queste sono le quattro rappresentazioni presenti nell'opera, le quali raffigurano le diverse sfaccettature del rapporto della Shelley con Godwin. Il dottor Frankenstein è il significante del rifiuto, è il padre di una creatura che ripudia e disprezza fin dal primo giorno:

Incapace di sopportare la vista dell'essere che avevo creato, mi precipitai fuori dal laboratorio, e per lungo tempo continuai a camminare avanti e indietro nella mia camera da letto, senza riuscire a calmare la mente e a prepararla al sonno (Shelley, 2019: 80).

(...) Sia maledetto il giorno in cui hai visto la luce diavolo detestabile! (...) Mi hai reso disgraziato oltre ogni dire. Non mi hai lasciato nessuna possibilità di riflettere se sono stato giusto o meno con te. Vattene! Liberami dalla vista della tua odiata figura! (Shelley, 2019: 136).

Tale odio paterno, lo percepisce anche lo stesso mostro: "Tu il mio creatore, mi detesti" (Shelley, 2019: 132). L'autrice però, descrivendo l'avversione che il dottore prova nei confronti della sua creatura, non si limita a proporre il naturale e conseguente istinto di vendetta che guiderà tutte le azioni del mostro, bensì lascia intendere qualcos'altro:

$\mathrm{Tu}$, il mio creatore, mi detesti; che speranza posso avere nei tuoi simili, che non $\mathrm{mi}$ devono nulla? Mi disprezzano e mi odiano. (...) eppure è in tuo potere risarcirmi, e liberarli da un male che solo tu puoi rendere così grande da inghiottire nei vortici della sua rabbia non solo te e la tua famiglia, ma migliaia di altri. Lasciati muovere a compassione e non disprezzarmi. (...) Tu mi accusi di omicidio; eppure distruggeresti la tua stessa creatura con la coscienza tranquilla (Shelley, 2019: 132).

Il mostro, nonostante compia omicidi terribili, agli occhi del lettore appare come una vittima. Sicuramente la domanda che si pone ("tu, il mio creatore, mi detesti; che speranza posso avere nei tuoi simili, che non mi devono nulla?") ci offre la prima spiegazione possibile di tale fenomeno. Se suo padre, il suo creatore, la figura che dovrebbe amare incondizionatamente il proprio figlio, lo odia a tal punto da non poter "tollerare l'orrore di quel volto", come possono gli altri salvarlo da un'esistenza che appare destinata alla solitudine e all'odio? Come sostiene Hardisson (2020) la lotta di Frankenstein (come quella di Mary) è rivolta al riconoscimento della propria identità che gli viene negata. Questa privazione lo fa diventare un soggetto perverso in cui la malvagità è un effetto secondario della sofferenza della sua emarginazione.

Mary Shelley sembra voler sottolineare la responsabilità della figura paterna nei confronti di un'esistenza condannata alla miseria, quanto sia forgiante la modalità con la quale il padre trasmette al figlio il proprio amore e quanto ne condizioni 
"l'impalpabile essenza del soggetto uomo, il suo fragile posto nel tempo" (Brunetti, 2003: 6).

In tutto il corso dell'opera il dottor Frankenstein pare ignorare questa sua responsabilità, ma quando il mostro lo implora di ascoltare la sua storia, lo scienziato confessa: "Per la prima volta, inoltre, sentii quali fossero i doveri di un creatore nei riguardi della sua creatura, e capii che avrei dovuto renderlo felice prima di lamentarmi della sua malvagità" (Shelley, 2019: 136).

Oltrel'incapacità degli altri di accettare la creatura a causa del mancato amore paterno, la ripugnanza del dottor Frankenstein verso il mostro condiziona l'impossibilità di amare della creatura stessa, proprio perché è assente l'insegnamento del concetto di amore. Quando il mostro guarda i gesti d'affetto degli abitanti della casa del bosco, si chiede spesso cosa fossero quelle tenerezze, da cosa dipendessero e soprattutto il motivo per il quale lui ne era privo.

L'opera sottolinea quanto, nonostante la mancata accettazione paterna, l'immagine del figlio sia legata al suo creatore, facendo sì che il lettore identifichi il dottore come il responsabile delle disgrazie della creatura. Non riuscendo a capacitarsi del perché lui fosse così ripugnante rispetto al resto degli uomini, il mostro fa ricadere la responsabilità sullo scienziato:

Maledetto creatore! Perché hai dato forma a un mostro così orrendo che perfino tu ti sei allontanato da me in preda al disgusto? Dio, nella sua misericordia, ha creato l'uomo bello e affascinante, a sua propria immagine; ma la mia figura è una copia oscena della tua, resa ancora più orribile proprio dalla somiglianza (Shelley, 2019: 136).

È proprio in questi termini che Frankenstein appare una forte dichiarazione sull'immoralità di costringere alla vita un'entità che non è stata equipaggiata per essere all'altezza di tale compito.

\section{Conclusioni}

Frankenstein di Mary Shelley ci appare una riflessione largamente indirizzata sul padre, tanto a livello di contenuto come di personaggi e schemi narrativi. Si tratta di un testo completamente privo di figure femminili che problematizza il rapporto padre/ figlia, dove la una situazione di abbandono da parte del padre, consegue la ricerca del figlio della figura paterna. Mary Shelley si traveste da uomo e si cala nei panni del mostro per impersonare questa ricerca. Significativamente, non avendo un nome, anche il mostro è dotato di una certa ambivalenza e può diventare lui stesso figura dell'androgino e simbolo di un'identità incompiuta, proprio a causa del mancato riconoscimento da parte del padre. 
Simbolicamente la storia del mostro traspone autobiograficamente la relazione di Mary con suo padre, che fin dall'infanzia delegò ad altri l'educazione della figlia. La rappresentazione dell'amore-odio fra padre e figlio in Frankenstein riflette il suo difficile rapporto con il genitore, nel quale si affaccia l'ombra dell'incesto e l'ideale androgino che rappresenta la figlia, in quanto scrittrice che accede a un mondo maschile.

\section{RIFERIMENTI BIBLIOGRAFICI}

Bolen, J. S., Le dee dentro la donna, Roma, Astrolabio Ubaldini editore, 1991.

Botting, F., Mary Shelley, Frankenstein, New York, St. Martin's, 1995.

Brown, W., The life of William Godwin, New York, 1926.

Brunetti, B., La figura del padre e la scrittura letteraria, Università degli studi di Bari, Milano, 2003.

Castellano, I. G. “El monstruo como alegoría de la mujer autora en el Romanticismo: Frankenstein y Sab", Revista hispánica moderna 60.2 (2007), pp. 187-203.

Chaves, J. R., “Incestuosa Madame Frankenstein”, Anuario de Letras Modernas 11 (2003), pp. 117-120.

Darrow, M., Revolution in the house: Family, Class and Inheritance in Southern France, 1775-1825, Princeton, Princeton University Press, 1989.

Felski, R., Literature after Feminism, Chicago, University of Chicago Press, 2003.

Ferré, R., "Frankenstein: una versión política del mito de la maternidad." Debate feminista, n. 6 (1992), pp. 32-43.

Foucault, M., The Order of Things, London, Tavistock, 1970.

Gilbert, S. M., and Gubar, S., The Mad Woman in the Attic: The Woman Writer and the Nineteenth Century Literary Imagination, New Heaven, Yale University Press, 1979.

Godwin, W., Enquiry Concerning Political Justice and it's influence, general virtue and happiness by W. Godwin, 2 vol., G.C. And J. Robinson, Paternoster, Row, 1793.

Goffman, E., Estigma. La identidad deteriorada, Buenos Aires. Amorrortu, 1989.

Hardisson Rumeu, Ana. "La Criatura de Frankenstein y la lucha por el reconocimiento", Isegoría 26 (2002), pp. 251-256.

Johnson, B., "My Monster / My Self". Modern Critical Interpretations. Mary Shelley 's Frank-enstein, Ed. Harold Bloom, New York, Chelsea, 1987.

Kirkpatrick, S., Las románticas: women writers and subjectivity in Spain, 1835-1850. Berkeley, University of California Press, 1989.

Madame de Stael, Corinna, Milano, Mondadori, 2006.

Marino, E., My Hideous progeny: padri e figli in Frankenstein di Mary Shelley, in Padri e Figli, Università di Roma Tor Vergata, 2009. 
Parrini, R., “Los poderes del padre: paternidad y subjetividad masculina.” J. Olavarría \& R. Parrini (Comp.), Masculinidades: Identidad, sexualidad y familia. Primer encuentro de masculinidad (2000): 69-78.

Pateman, C. "Feminist Critiques of the Public/Private Dichotomy." Public and Private in Social Life. Eds. Stanley I. Ben and Gerald F. Gaus, New York, St. Martin's Press, 1983.

Poovey, M., “'My Hideous Progeny': The Lady and the Monster”. Modern Critical Interpretations. Mary Shelley 's Frankenstein. Ed. Harold Bloom. New York, Chelsea, 1987, pp. 81-106.

Praz, M., Introduzione a Mary Shelley Frankenstein, Milano, Rizzoli, 2011, pp. 9-14.

Sapegno, M., Figlie del padre, Feltrinelli, Milano, 2018.

Shelley, M., Frankenstein, BUR Rizzoli, 2019.

Veeder, W., Mary Shelley and Frankenstein: The Fate of Androgyny, Chicago, University of Chicago Press, 1986.

Wollstonecraft, M., Vindicación de los derechos de la mujer, Madrid, Cátedra, 1994. 